\title{
Band Structure of Highly Mismatched Semiconductor Alloys: Coherent Potential Approximation
}

\author{
J. Wu $u^{1,2}, W$. Walukiewicz ${ }^{2}$, and E. E. Haller ${ }^{2,3}$ \\ 1. Applied Science and Technology Graduate Group, University of California, \\ Berkeley, CA 94720 \\ 2. Materials Sciences Division, Lawrence Berkeley National Laboratory, \\ Berkeley, CA 94720 \\ 3. Department of Materials Science and Engineering, University of California, \\ Berkeley, CA 94720
}

The many-impurity Anderson model is applied to compound semiconductor alloys in which metallic anion atoms are partially substituted by highly electronegative atoms at low concentrations. The interaction between the localized states derived from the electronegative atoms and the Bloch states of the semiconductor matrix is treated in a single-site coherent potential approximation. The solution for the Green's function provides dispersion relations and broadenings for the conduction band states. The calculations validate the dispersion relations previously obtained from the two-level band anticrossing model. The restructured dispersion relations and optical absorption coefficient are calculated and compared with experimental results of $\mathrm{GaAs}_{1-x} \mathrm{~N}_{x}$ alloys.

Electronic mail address: ﹎.walukiewicz@lbl.gov

PACS numbers: 71.20.Nr, 78.40.Fy, 71.10.Fd, 71.23.An 


\section{Introduction}

Over the past several decades, the physics of randomly disordered crystals has been studied extensively. An especially intense effort has been directed towards understanding of the electronic structure of random semiconductor alloys. One of the simplest treatments of such alloys is based on the Virtual Crystal Approximation (VCA) that is applicable to perfectly random alloys [1-2]. In this approximation, the electronic properties of the alloys are given by the linear interpolation between the properties of the end-point materials. The alloy disorder effects are typically included through a bowing parameter that describes the deviations from the VCA. The description of the composition dependence of the band gap in terms of the bowing parameter has been commonly used in a large variety of semiconductor alloys. It should be emphasized, however, that this approximation is expected to work reasonably well only for systems with bowing parameters much smaller than the energy gap.

Most of the studies of semiconductor alloy systems are restricted to the cases where there are only small differences between properties of the end-point semiconductor materials. Such "well-matched" alloys can be easily synthesized and their properties are close to the VCA predictions. Recent progress in the epitaxial growth techniques has led to successful synthesis of semiconductor alloys composed of materials with distinctly different properties. The properties of these "highly mismatched" alloys (HMAs) drastically deviate from the linear predictions of the VCA. The most prominent class of HMAs comprises the III- $\mathrm{V}_{1-x}-\mathrm{N}_{x}$ alloys, in which electronegative nitrogen substitutes group V anions in standard group III-V compounds. One of the striking effects of nitrogen incorporation into III-V semiconductors is a dramatic reduction in the fundamental band gap. A band gap reduction of more than 180 meV has been observed in $\mathrm{GaAs}_{1-x} \mathrm{~N}_{x}$ alloys with only $1 \% \mathrm{~N}$ [3]. Similar effects were observed in $\mathrm{GaP}_{1-x} \mathrm{~N}_{x}$ [4], $\operatorname{InP}_{1-x} \mathrm{~N}_{x}$ [5], $\mathrm{GaSb}_{y} \mathrm{As}_{1-x-y} \mathrm{~N}_{x}$ [6] and $\mathrm{InSb}_{1-x} \mathrm{~N}_{x}$ [7] alloys. The large band gap bowing and the lower-than-usual band gap pressure dependence have been found also in group II-VI HMAs such as $\mathrm{ZnTe}_{1-x} \mathrm{~S}_{x}$ and $\mathrm{ZnTe}_{1-x} \mathrm{Se}_{x}$ [8], where electronegative $\mathrm{S}$ or Se substitute metallic Te atoms. 
The energy band structure of HMAs has been explained in terms of the Band Anticrossing (BAC) model [8-9]. The model accurately describes the composition and pressure dependencies of the fundamental band gaps of HMAs. Furthermore, it predicted several new effects such as a $\mathrm{N}$-induced enhancement of the electron effective mass [10] and an improvement in the donor activation efficiency [11] in $\operatorname{In}_{y} \mathrm{Ga}_{1-y} \mathrm{As}_{1-x} \mathrm{~N}_{x}$ alloys, and the change in the nature of the fundamental band gap from indirect to direct in $\mathrm{GaP}_{1-x} \mathrm{~N}_{x}$ [12]. All these predictions have now been confirmed experimentally.

In the BAC model, the restructuring of the conduction band is a result of an anticrossing interaction between highly localized $\mathrm{A}_{1}$ states of the substitutional $\mathrm{N}$ atoms and the extended states of the host semiconductor matrix. The interaction between these two types of states has been treated in the simplest possible manner that does not account for expected severe level broadening effects. These effects profoundly affect the line shape of the observed optical transitions and they entirely dictate energy dissipative processes such as free carrier transport.

To address these issues and to put the BAC model on a firmer theoretical base, we have adopted the many-impurity Anderson model that has been widely used to treat the interaction between impurity states and band states. The original Anderson model has been developed to describe a single impurity atom of a transition metal or a rare-earth element in a non-magnetic metal. In Anderson's $s-d$ exchange model [13], the electron system is separated into a delocalized part of the matrix metal which is described in terms of the band theory, and a localized level of the $d$ shell electrons of the transition metal impurity atom. A dynamical mixing term is introduced into the Hamiltonian of the system to describe the hybridization between the band states and the localized impurity states. Solving the Anderson Hamiltonian it has been found that, as a result of the hybridization, the impurity $d$ state becomes a virtual energy state with an imaginary energy part proportional to the strength of the $s-d$ hybridization. The imaginary part of the eigen-energy of the virtual state defines the width of the density distribution of the $d$ state, and determines the lifetime of the state before the $d$ electrons are delocalized into the band states through the exchange interaction. Self-consistency calculations can be performed to find the conditions for the existence of localized magnetic moments. 
The single impurity Anderson model has been extended to explain the properties of cerium-based heavy-Fermion systems. A periodic coherent Anderson model has been developed and investigated over the years to accommodate both the spatial periodicity and the localization of the $4 f$ orbitals in the systems $[14,15]$. The energy dispersion of the system is restructured into two subbands, a result of the hybridization between the localized orbitals and the band states. The newly-generated indirect gap between the subbands has profound effects on the electrical and thermodynamical properties of the system [16].

A many-impurity Anderson model has been proposed to describe the electronic properties of semiconductor crystals with low concentrations of deep-level transitionmetal impurities [17-18]. Unlike ordinary hydrogenic impurity states in semiconductors, these impurity states are characterized by two independent parameters: the spatial extension of their wave function and their energy level with respect to the nearest band edge of the host. The phase diagram for the electronic properties of crystals with such impurities is much richer than for the simple hydrogen-like impurities. For example, as the impurity concentration increases, in addition to the trend of conductivity increasing as a result of the Mott transition [19], hybridization between the impurity states and the band states of the host can considerably suppress the conductivity of the system in the form of inter-state electron scattering. Therefore, transport properties of the crystal are diversified by the competition between these two opposite processes.

In this paper, we use the many-impurity Anderson model to evaluate the interaction between the randomly distributed localized states and the extended states in HMAs. We solve this problem within the single site coherent potential approximation (CPA). The calculations reproduce the BAC model results for the restructuring of the conduction band. The imaginary part of the Green's function also yields new information on the electronic level broadening that is used to determine the broadening of the optical transitions and to calculate the free electron mobility.

\section{Theory}


It has been predicted by Hjalmarson et. al. that incorporation of isoelectronic impurities into semiconductors gives rise to highly localized levels [20]. The energy of these levels depends on the electronegativity of the substitutional impurity. In the case of highly electronegative impurities substituting metallic anions in compound semiconductors, the energy levels are located close to the conduction band edge [20]. For example, substitutional nitrogen as an isoelectronic impurity in GaAs generates an $\mathrm{A}_{1}$ symmetry localized level resonant with the conduction band of GaAs at $\sim 0.23 \mathrm{eV}$ above the conduction band edge. This resonant level has been observed in optical experiments when the level is moved into the GaAs band gap by applying hydrostatic pressure [21] or alloying with $\mathrm{GaP}$ [22]. With increasing $\mathrm{N}$ impurity concentration, the interaction between the localized $\mathrm{N}$ levels and the GaAs band states alters the electronic structure of the resulting $\operatorname{GaAs}_{1-x} \mathrm{~N}_{x}$ system.

We describe the electronic structure of HMAs (e.g., $\operatorname{GaAs}_{1-x} \mathrm{~N}_{x}$ ) by considering an interaction between the localized and extended states within the many-impurity Anderson model. The total Hamiltonian of the system is the sum of three terms [17, 18],

$H=\sum_{k} E_{k}^{c} c_{k}^{+} c_{k}+\sum_{j} E_{j}^{d} d_{j}^{+} d_{j}+\frac{1}{\sqrt{N}} \sum_{j, k}\left(e^{i k \cdot j} V_{k j} c_{k}^{+} d_{j}+\right.$ h.c. $)$,

where the first term is the Hamiltonian of the electrons in the band states with energy dispersion $E_{k}^{c}$, and the second term corresponds to the energy of the electron localized on the $j$ th impurity site with energy $E_{j}^{d}$. To simplify the expressions, we use a vector $\boldsymbol{j}$ to denote the 3-dimentional coordinates of the $j$ th site. The third term describes the change in the single electron energy due to the dynamical mixing between the band states and the localized states. It is assumed that only one band and one impurity level are involved in the process. Following Anderson's scheme, the hybridization strength is characterized by the parameter $V_{k j}$ defined by the following equation [13],

$$
\begin{aligned}
\left\langle\boldsymbol{k}\left|H_{H F}\right| d\right\rangle & =\frac{1}{\sqrt{N}} \sum_{l, j} e^{i \boldsymbol{k} \cdot \boldsymbol{l}} \int a^{*}(\boldsymbol{r}-\boldsymbol{l}) H_{H F}(\boldsymbol{r}) \varphi_{d}(\boldsymbol{r}-\boldsymbol{j}) d \boldsymbol{r} \\
& =\frac{1}{\sqrt{N}} \sum_{j} e^{i \boldsymbol{k} \cdot \boldsymbol{j}} \cdot \sum_{l} e^{i \boldsymbol{k}(\boldsymbol{l}-j)} \int a^{*}(\boldsymbol{r}-\boldsymbol{l}) H_{H F}(\boldsymbol{r}) \varphi_{d}(\boldsymbol{r}-\boldsymbol{j}) d \boldsymbol{r} \\
& \equiv \frac{1}{\sqrt{N}} \sum_{j=1} e^{i \boldsymbol{k} \cdot \boldsymbol{j}} V_{\boldsymbol{k} \boldsymbol{j}},
\end{aligned}
$$


where $a(\boldsymbol{r}-\boldsymbol{j})$ and $\varphi_{d}(\boldsymbol{r}-\boldsymbol{j})$ are the Wannier function belonging to the band and the localized wavefunction of the impurity on the $j$ th site, respectively. $H_{H F}(\boldsymbol{r})$ is the single electron energy described in the Hartree-Fock approximation [13].

The Fourier transform of the retarded Green's function, $G_{\boldsymbol{k} \boldsymbol{k}^{\prime}}(E)=<<c_{\boldsymbol{k}} \mid c_{\boldsymbol{k}^{\prime}}^{+}>>$, satisfies the following equation of motion [23-24],

$E<<c_{k}\left|c_{\boldsymbol{k}^{\prime}}^{+}>>=<\left[c_{\boldsymbol{k}}, c_{\boldsymbol{k}^{\prime}}^{+}\right]_{+}>+<<\left[c_{k}, H\right]\right| c_{\boldsymbol{k}^{\prime}}^{+}>>$.

In Eq.(3), the bracket $\langle\cdots\rangle$ represents the ensemble average. As follows from the commutation relations between the operators, an integral equation for $G_{k k}$, has the form,

$$
G_{k k^{\prime}}=\delta_{k k^{\prime}} G_{k \boldsymbol{k}}^{(0)}+\frac{1}{N} G_{k \boldsymbol{k}}^{(0)} \sum_{\boldsymbol{k}^{\prime \prime} j} \widetilde{V} \cdot e^{i\left(\boldsymbol{k}-\boldsymbol{k}^{\prime \prime)}\right) \cdot j} G_{\boldsymbol{k}^{\prime \prime} \boldsymbol{k}^{\prime}}
$$

where $G_{\boldsymbol{k} \boldsymbol{k}^{\prime}}^{(0)}=\delta_{\boldsymbol{k} \boldsymbol{k}^{\prime}}\left(E-E_{\boldsymbol{k}}^{c}+i 0^{+}\right)^{-1}$ is the unperturbed Green's Function, and the renormalized interaction parameter is given by

$$
\widetilde{V}=V_{\boldsymbol{k} j} \cdot V_{\boldsymbol{k}^{\prime \prime} j} /\left(E-E_{j}^{d}\right) \approx V^{2} /\left(E-E_{j}^{d}\right),
$$

where $V$ is the average value of $V_{k j}$, assuming weak dependencies on $\boldsymbol{k}$ and $\boldsymbol{j}$. The justification of this assumption will be discussed later.

For the single impurity case, we can set $\boldsymbol{j}=0$. The equation chain represented by Eq.(4) is closed and has the following solution,

$$
G_{k k^{\prime}}=\delta_{k k^{\prime}} G_{k k}^{(0)}+\frac{\widetilde{V}}{N} G_{k k}^{(0)} G_{k^{\prime} k^{\prime}}^{(0)}\left[1-\frac{\widetilde{V}}{N} \sum_{k^{\prime \prime}} G_{k^{\prime \prime} k^{\prime \prime}}^{(0)}\right]^{-1} .
$$

Meanwhile,

$$
\sum_{k^{\prime \prime}} G_{k^{\prime \prime} k^{\prime \prime}}^{(0)}=\sum_{k^{\prime \prime}} \frac{1}{E-E_{k^{\prime \prime}}^{c}+i 0^{+}} \approx i \pi \beta N \rho_{0}\left(E^{d}\right)
$$

where $\rho_{0}(\varepsilon)$ is the unperturbed density of states of $E_{k}^{c}$ per unit cell. Since $\rho_{0}(\varepsilon)$ only weakly depends on energy, we can assume that it is constant in first order approximation, with an effective value equal to the unperturbed density of states evaluated at $E^{d}$ and multiplied by a prefactor $\beta$. Using this approximation, Eq.(6) gives the eigen-energies for 
the system, $E=E_{k}^{c}$ and $E=E^{d}+i \pi \beta V^{2} \rho_{0}\left(E^{d}\right)$, which are the solutions of the original single-impurity Anderson model [13].

In general, we shall consider finite but dilute concentrations of impurities, $0<x<<1$. We assume that the impurities are randomly distributed in space, so that we can carry out a configurational averaging, neglecting correlations between positions of the impurities. In this case, the single-site coherent potential approximation (CPA) is adequate for the many-impurity system [25-26]. In the CPA, consecutive multiple scatterings from each single impurity atom are fully taken into account, but correlations between scatterings from different impurity atoms are neglected due to the lack of coherence between the randomly distributed impurity sites. The CPA treatment leads to the result that $[17,25-26]$, after the configurational averaging, the average Green's function partially restores the space translational invariance, and $\boldsymbol{k}$ resumes its welldefined properties as a good quantum number. In momentum space, the diagonal Green's function in CPA can be written as [17,25-26]

$$
G_{k \boldsymbol{k}}(E)=\left[E-E_{k}^{c}-\sigma(E)\right]^{-1}
$$

where the average self-energy is proportional to the impurity concentration,

$$
\sigma(E)=\frac{x \widetilde{V}}{1-(\widetilde{V} / N) \sum_{k} G_{k \boldsymbol{k}}} \equiv \frac{x \widetilde{V}}{1-\widetilde{V} G(E)} .
$$

The average Green's function $G(E)$ defined in Eq.(9) is determined by the selfconsistency equation,

$$
G(E)=\frac{1}{N} \sum_{\boldsymbol{k} \in B Z} \frac{1}{E-E_{\boldsymbol{k}}^{c}-x \widetilde{V} /[1-\widetilde{V} G(E)]} .
$$

Noting that, as in the case of Eq.(7), the imaginary part of the denominator in Eq.(10) is small (in proportion to a small number $x$ ), we can replace Eq.(10) by Eq.(7) as the lowest order approximation. Eq.(8) thus becomes

$$
G_{k \boldsymbol{k}}(E)=\left[E-E_{k}^{c}-\frac{V^{2} x}{E-E^{d}-i \pi \beta V^{2} \rho_{0}\left(E^{d}\right)}\right]^{-1} .
$$

The new dispersion relations are determined by the poles of $G_{\boldsymbol{k}}(E)$, and the solutions are given by an equivalent two-state-like eigen-value problem, 
$\left|\begin{array}{cc}E_{\boldsymbol{k}}^{c}-E(\boldsymbol{k}) & V \sqrt{x} \\ V \sqrt{x} & E^{d}+i \Gamma_{d}-E(\boldsymbol{k})\end{array}\right|=0$,

where $\Gamma_{d}=\pi \beta V^{2} \rho_{0}\left(E^{d}\right)$ is the broadening of $E^{d}$ in the single-impurity Anderson Model. If $\Gamma_{d}=0$, Eq.(12) is reduced to the BAC model [9] with two restructured dispersions for the upper and lower conduction subbands,

$E_{ \pm}(\boldsymbol{k})=\frac{1}{2}\left\{\left(E_{\boldsymbol{k}}^{c}+E^{d}\right) \pm \sqrt{\left(E_{\boldsymbol{k}}^{c}-E^{d}\right)^{2}+4 V^{2} x}\right\}$.

If the broadening $\Gamma_{d}$ is nonzero but small, so that $2 V \sqrt{x}>>\pi \beta V^{2} \rho_{0}\left(E^{d}\right)$ and $\left|E_{k}^{c}-E^{d}\right|>>\pi \beta V^{2} \rho_{0}\left(E^{d}\right)$, we obtain an approximate analytical solution for Eq.(12),

$\widetilde{E}_{ \pm}(\boldsymbol{k}) \approx E_{ \pm}(\boldsymbol{k})+i \Gamma_{d} \frac{\left[E_{ \pm}(\boldsymbol{k})-E_{k}^{c}\right]}{\left[E_{ \pm}(\boldsymbol{k})-E_{\boldsymbol{k}}^{c}\right]+\left[E_{ \pm}(\boldsymbol{k})-E^{d}\right]} \equiv E_{ \pm}(\boldsymbol{k})+i \Gamma_{ \pm}(\boldsymbol{k})$,

where $E_{ \pm}(\boldsymbol{k})$ is defined in Eq.(13). The imaginary part of the dispersion relations defines the hybridization-induced uncertainty of the energy. We note that the imaginary part in Eq.(14) is proportional to the admixture of the localized states to the restructured wavefunctions in the two-state-like-perturbation picture described by Eq.(12),

$\Gamma_{ \pm}(\boldsymbol{k})=\left|\left\langle\varphi_{d} \mid E_{ \pm}(\boldsymbol{k})\right\rangle\right|^{2} \cdot \Gamma_{d}$.

\section{Discussions and Comparison with Experimental Results}

As an example, Fig.1 shows the dispersion relations given by Eq.(13) for $\operatorname{GaAs}_{0.995} \mathrm{~N}_{0.005}$ near the Brillouin zone center. The broadening of the dispersion relations is given by the imaginary part of Eq.(14). In the calculation, the hybridization parameter $V=2.7 \mathrm{eV}$, taken to be an experimentally-determined constant [9]. The density of states for the GaAs conduction band edge is assumed to have a parabolic form following the effective-mass theory,

$\rho_{0}(\varepsilon)=4 \pi \sqrt{\varepsilon-E_{0}^{c}} / \varepsilon_{B}^{3 / 2}$, 
where $\varepsilon_{B}=\hbar^{2}(2 \pi / b)^{2} /\left(2 m^{*}\right)$ is of the order of the conduction band width. $b=5.65 \AA$ is the lattice constant of the unit cell, and $m^{*}=0.067 m_{0}$ is the electron effective mass of GaAs. The prefactor $\beta$ is taken to be equal to 0.22 , as will be estimated below.

The density of states can be calculated from the imaginary part of the Green's function and is given by the expression,

$$
\rho(E)=\frac{1}{\pi} \operatorname{Im} \sum_{k} G_{k k}(E)=\frac{1}{\pi} \int \rho_{0}\left(E_{k}^{c}\right) \operatorname{Im}\left[G_{k k}(E)\right] d E_{k}^{c} .
$$

The integration converges rapidly with $E_{\boldsymbol{k}}^{c}$ in a small range that is in proportion to $x$. The calculated perturbed density of states for $\mathrm{GaAs}_{1-x} \mathrm{~N}_{x}$ with several small values of $x$ is shown in Fig.2. Note that the anticrossing interaction leads to a dramatic redistribution of the electronic states in the conduction band. The most striking feature of the density of states curves is the clearly seen gap between $E_{+}$and $E_{-}$that evolves with increasing $\mathrm{N}$ content.

In order to illustrate the effect of the state broadening on the optical properties, we consider the spectral dependence of the interband absorption in $\operatorname{In}_{y} \mathrm{Ga}_{1-y} \mathrm{As}_{1-x} \mathrm{~N}_{x}$ alloys. The optical absorption coefficient due to the transitions from the valence bands to the restructured conduction bands can be written in the form of the joint density of states as

$\alpha(E) \propto \frac{1}{E} \sum_{v} \int \rho_{0}\left(E_{k}^{c}\right) \operatorname{Im}\left[G_{k k}\left(E+E_{k}^{v}\right)\right] d E_{k}^{c}$.

In this expression, the sum over $v$ represents the sum of the contributions from the heavyhole, the light-hole, and the spin-orbital split-off valence bands. Assuming parabolic forms for the dispersions of the valence bands, $E_{k}^{v}$, near the Brillouin zone center, we have calculated the optical absorption for $\operatorname{In}_{0.04} \mathrm{Ga}_{0.96} \mathrm{As}_{0.99} \mathrm{~N}_{0.01}$ for which experimental results are available [27]. The comparison between the calculation and the experimental data is shown in Fig.3. In the calculation, the only parameter that has been adjusted is the prefactor $\beta$ used to scale the energy broadening, and the best fitting with the experimental data is obtained with $\beta=0.22$. The calculation clearly reproduces the two bumps on the absorption curve, i.e., one starting at $\sim 1.8 \mathrm{eV}$ due to the onset of the transitions from the heavy-hole and light-hole valence bands to $E_{+}$, and one starting at $\sim 1.5 \mathrm{eV}$ due to the onset of the transition from the split-off valence band to $E_{\text {. }}$. The more rapid rise of the 
experimental data at the absorption edge near $1.2 \mathrm{eV}$ is most likely due to continuum exciton absorption effect, which is not considered in the calculation.

According to the band anticrossing Eq.(13), the hybridization results in a energy gap near $E^{d}$ with magnitude depending on $x$,

$$
\Delta=E_{+}(0)-E^{d}=\frac{1}{2}\left(\sqrt{\left(E^{d}-E_{0}^{c}\right)^{2}+4 V^{2} x}-\left(E^{d}-E_{0}^{c}\right)\right) \approx \frac{V^{2} x}{\left|E^{d}-E_{0}^{c}\right|} .
$$

The broadening of $\widetilde{E}_{ \pm}(k)$ near the edge of the small gap is approximately $\Gamma_{d}=\pi \beta V^{2} \rho_{0}\left(E^{d}\right)$. To have a well-defined band restructuring, the gap between the two subbands should be larger than the energy uncertainty, i.e., $\Delta>\Gamma_{d}$. This condition gives a lower limit of $x$ for the band restructuring,

$$
x>x_{c} \equiv 4 \pi^{2} \beta\left(\left|E^{d}-E_{0}^{c}\right| / \varepsilon_{B}\right)^{3 / 2} \text {. }
$$

For $\mathrm{N}$ in GaAs, this concentration limit is $x_{c} \sim 0.0016$.

The band broadening function in Eq.(15) defines the lifetime of the free electrons through the uncertainty principle. The mean free path of the electrons is the distance that the free electrons on the Fermi surface travel within the lifetime. The upper limit of $x$ for the applicability of CPA treatment can be set as the concentration for which the average distance between impurity atoms is equal to the mean free path. The upper limit of $x$ is thus inversely proportional to the free electron concentration, linked by the Fermi velocity.

In the Green's function calculation, the $\boldsymbol{k}$ dependence of $V_{\boldsymbol{k} j}$ is assumed to be weak on the momentum scale we are interested in. In Eq.(5), the parameter $V_{\boldsymbol{k} j}$ is averaged over the impurity sites and in $\boldsymbol{k}$ space. In the simplest case, all the impurity atoms are of the same type, so that the $\boldsymbol{j}$ dependence of $V_{\boldsymbol{k}}$ is removed. The $\boldsymbol{k}$ dependence of $V_{k j}$ can be estimated from Eq.(2). Assuming that the Hartree-Fock energy varies slowly in space and can be replaced by a constant $\varepsilon_{H F}$, we have

$$
V_{k}=\varepsilon_{H F} \cdot \sum_{l} e^{i \boldsymbol{k} \cdot \boldsymbol{l}} \int a^{*}(\boldsymbol{r}-\boldsymbol{l}) \varphi_{d}(\boldsymbol{r}) d \boldsymbol{r} .
$$

Due to the localized character of both $a(r)$ and $\varphi_{d}(\boldsymbol{r})$, the overlap integral in Eq.(21) is essentially zero when $a(\boldsymbol{r})$ and $\varphi_{d}(\boldsymbol{r})$ are located on two sites far apart from each other. 
In an attempt to model the $\boldsymbol{k}$-dependence of $V_{\boldsymbol{k}}$, we replace the integral in Eq.(21) by an exponentially decaying function $\sim \exp \left(-l / l_{d}\right)$, and obtain

$$
V_{k}=\frac{V_{0}}{\left(1+l_{d}^{2} k^{2}\right)^{2}} .
$$

There is experimental evidence indicating that the values of $V_{k}$ at the $\mathrm{L}$ point in $\mathrm{GaAs}_{1-}$ ${ }_{x} \mathrm{~N}_{x}$ [28] and at the $\mathrm{X}$ point in $\mathrm{GaP}_{1-x} \mathrm{~N}_{x}$ [29] are about 3 4 times smaller than the $V_{\boldsymbol{k}}$ at the $\Gamma$ point. This ratio corresponds to a wavefunction decay length of the order of the lattice constant, $l_{d} \sim b$. This result indicates that the off-zone-center conduction band minima are affected by the anticrossing interaction only when their energies are close to the localized level. This is consistent with recent measurements of the optical properties of $\mathrm{In}_{y} \mathrm{Ga}_{1-y} \mathrm{As}_{1-x} \mathrm{~N}_{x}$ alloys, which have shown that alloying with $\mathrm{N}$ has only very small effects on the high energy transitions at large $\boldsymbol{k}$ vectors [30].

\section{Conclusions}

In summary, we have applied the many-impurity Anderson model to the electronic structure of highly mismatched semiconductor alloys. The band restructuring and energy broadening effects are investigated within the coherent potential approximation. The dispersion relations calculated using the coherent potential approximation reproduce the results of the two-level band anticrossing model [9]. The band restructuring leads to a strongly perturbed density of states for the conduction band. We show that the conduction band restructuring and the energy broadening have to be included to explain the spectral dependence of the absorption coefficient of $\operatorname{In}_{y} \mathrm{Ga}_{1-y} \mathrm{As}_{1-}$ ${ }_{x} \mathrm{~N}_{x}$ alloys. The energy broadening function can also be used to calculate the effects of the hybridization on the transport properties of these highly mismatched alloys.

We thank B. Cardozo for his help with the computational software. J. Wu acknowledges the Berkeley Fellowship from the University of California, Berkeley. The work at Lawrence Berkeley National Laboratory is part of the project on the "Photovoltaic Materials Focus Area" in the DOE Center of Excellence for the Synthesis and Processing of Advanced Materials, and was supported by the Director, Office of 
Science, Office of Basic Energy Sciences, Division of Materials Sciences of the U.S. Department of Energy under Contract No. DE-AC03-76SF00098. 


\section{REFERENCES}

[1] J. A. VanVechten and T. K. Bergstresser, Phys. Rev. B 1, 3351 (1970).

[2] R. Hill and D. Richardson, J. Phys. C 4, L289 (1971).

[3] M. Weyers, M. Sato and H. Ando, Jpn. J. Appl. Phys. 31, L853(1992); K. Uesugi, N. Marooka and I. Suemune, Appl. Phys. Lett. 74, 1254(1999).

[4] N. Baillargeon, K. Y. Cheng, G. F. Hofler, P. J. Pearah and K. C. Hsieh, Appl. Phys. Lett. 60, 2540(1992).

[5] W. G. Bi and C. W. Tu, J. Appl. Phys. 80, 1934(1996).

[6] J. C. Harmand, G. Ungaro, J. Ramos, E. V. K. Rao, G. Saint-Girons, R. Teissier, G. Le Roux, L. Largeau and G. Patriarche, J. Crystl. Growth, to be published.

[7] B. N. Murdin, M. Karmal-Saadi, A. Lindsay, E. P. O’Reilly, A. R. Adams, G. J. Nott, J. G. Crowder, C. R. Pidgeon, I. V. Bradley, J. P. R. Wells, T. Burke, A. D. Johnson and T. Ashley, Appl. Phys. Lett. 78, 1558(2001).

[8] W. Walukiewicz, W. Shan, K. M. Yu, J. W. Ager III, E. E. Haller, I. Miotkowski, M. J. Seong, H. Alawadhi and A. K. Ramdas, Phys. Rev. Lett. 85, 1552(2000).

[9] W. Shan, W. Walukiewicz, J. W. Ager III, E. E. Haller, J. F. Geisz, D. J. Friedman, J. M. Olson and S. R. Kurtz, Phys. Rev. Lett. 82, 1221(1999).

[10] M. Hettrich, M. D. Dawson, A. Yu Egorov, D. Bernklau and H. Riechert, Appl. Phys. Lett. 76, 1030(2000); J. Wu, W. Shan, W. Walukiewicz, K. M. Yu, J. W. Ager III, E. E. Haller, H. P. Xin and C. W. Tu, Phys. Rev. B 64, 85320(2001).

[11] K. M. Yu, W. Walukiewicz, W. Shan, J. W. Ager III, J. Wu, E. E. Haller, J. F. Geisz, D. J. Friedman and J. M. Olson, Phys. Rev. B 61, R13337 (2000).

[12] W. Shan, W. Walukiewicz, K. M. Yu, J. Wu, J. W. Ager III, E. E. Haller, H. P. Xin and C. W. Tu, Appl. Phys. Lett. 76, 3251 (2000).

[13] P. W. Anderson, Phys. Rev. 124, 41(1961).

[14] A. Yoshimori and H. Kasai, J. Magn. Magn. Mat. 31, 475(1983).

[15] P. A. Lee, T. M. Rice, J.W. Serene, L. J. Sham and J. W. Wilkins, Comments Cond. Mat. Phys. 12, 99(1986); C. M. Varma and Y. Yafet, Phys. Rev. B 13, 2950(1976).

[16] D. M. Newns and N. Read, Advances in Physics, 36, 799(1987); Peter S. Riseborough, Phys. Rev. B, 45, 13984(1992). 
[17] A. N. Kocharyan, Soc. Phys. Solid State 28(1), 6(1986).

[18] M. A. Ivanov and Yu. G. Pogorelov, Sov. Phys. JETP 49, 510(1979); M. A. Ivanov and Yu. G. Pogorelov, Sov. Phys. JETP 61, 1033(1985).

[19] N. F. Mott and E. A. Davis, Electronic Processes in Non-Crystalline Materials, Clarendon Press, Oxford (1971).

[20] Harold P. Hjalmarson, P. Vogl, D. J. Wolford and John D. Dow, Phys. Rev. Lett. 44, $810(1980)$.

[21] D. J. Wolford, J. A. Bradley, K. Fry, J. Thompson, in Physics of Semiconductors, ed. by J. D. Chadi and W. A. Harrison, Springer, New York 1984.

[22] W. Y. Hsu, J. D. Dow, D. J. Wolford, B. G. Streetman, Phys. Rev. B 16, 1597(1977).

[23] G. M. Zaslavskii, E. V. Kuz'min and I. S. Sandalov, Sov. Phys. JETP, 40, 707(1975); M. A. Ivanov and Yu. G. Pogorelov, Sov. Phys. Solid State, 16, 2140(1975).

[24] S. Doniach and E. H. Sondheimer, Green's Functions for Solid State Physics, W. A. Benjamin, Inc. 1974.

[25] Fumiko Yonezawa and Kazuo Morigaki, Supplement of the Progress of Theoretical Physics, No.53, (1973).

[26] R. J. Elliott, J. A. Krumhansl and P. L. Leath, Rev. Mod. Phys. 46, 465(1974).

[27] P. Perlin, P. Wisniewski, C. Skierbiszewski, T. Suski, E. Kaminska, S. G. Subramanya, E. R. Weber, D. E. Mars and W. Walukiewicz, Appl. Phys. Lett. 76, 1279 (2000).

[28] J. D. Perkins, A. Masarenhas, J. F. Geisz and D. J. Friedman, Phys. Rev. B 64, 121301 (2001).

[29] J. Wu, W. W. Walukiewicz, K. M. Yu, J. W. Ager III, E. E. Haller, Y. Hong, H. P. Xin and C. W. Tu, to be published.

[30] C. Skierbiszewski, P. Perlin, P. Wisniewski, T. Suski, J. F. Geisz, K. Hingerl, W. Jantsch, D. Mars and W. Walukiewicz, Phys. Rev. B, to be published. 


\section{FIGURE CAPTIONS}

Fig.1 Conduction band restructuring according to Eq.(14) for $\operatorname{GaAs}_{0.995} \mathrm{~N}_{0.005}$. The broadening of the dispersion curves of the newly-formed subbands illustrates the energy uncertainties defined in Eq.(15). All the energies are referenced to the top of the valence band of GaAs.

Fig.2 Density of states of $\operatorname{GaAs}_{1-x} \mathrm{~N}_{x}$ alloys for a range of values of $x$ as compared with the unperturbed density of states. The two black dots on each curve indicate the energy positions of the $E_{-}$and $E_{+}$subband edges.

Fig.3 Calculated optical absorption coefficient in comparison with room-temperature experimental data for free-standing $\operatorname{In}_{0.04} \mathrm{Ga}_{0.96} \mathrm{As}_{0.99} \mathrm{~N}_{0.01}$. The oscillations below the absorption edge are due to Fabry-Perot interference. 


\section{FIGURES}

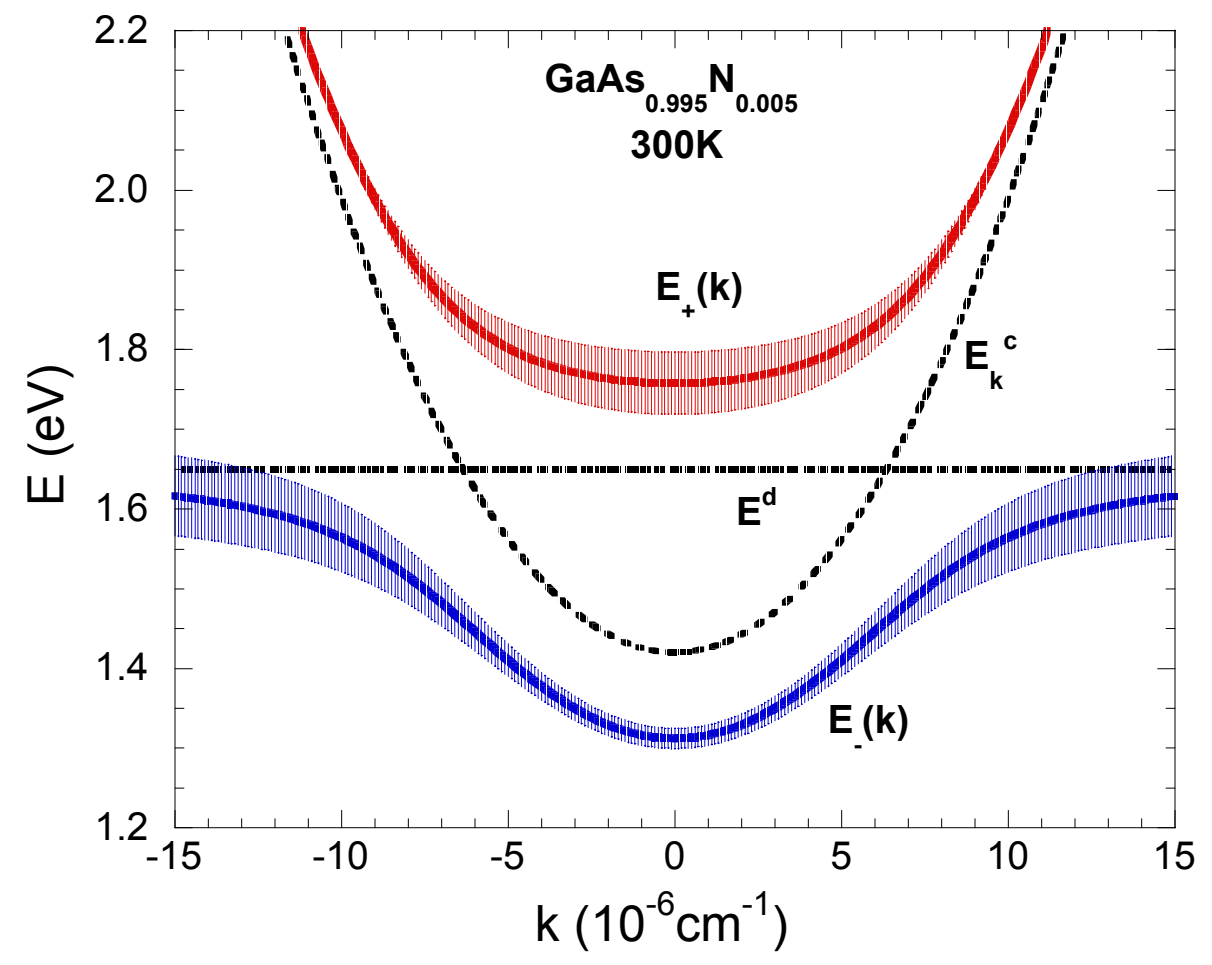

Fig. 1 of 3

J. Wu et. al. 


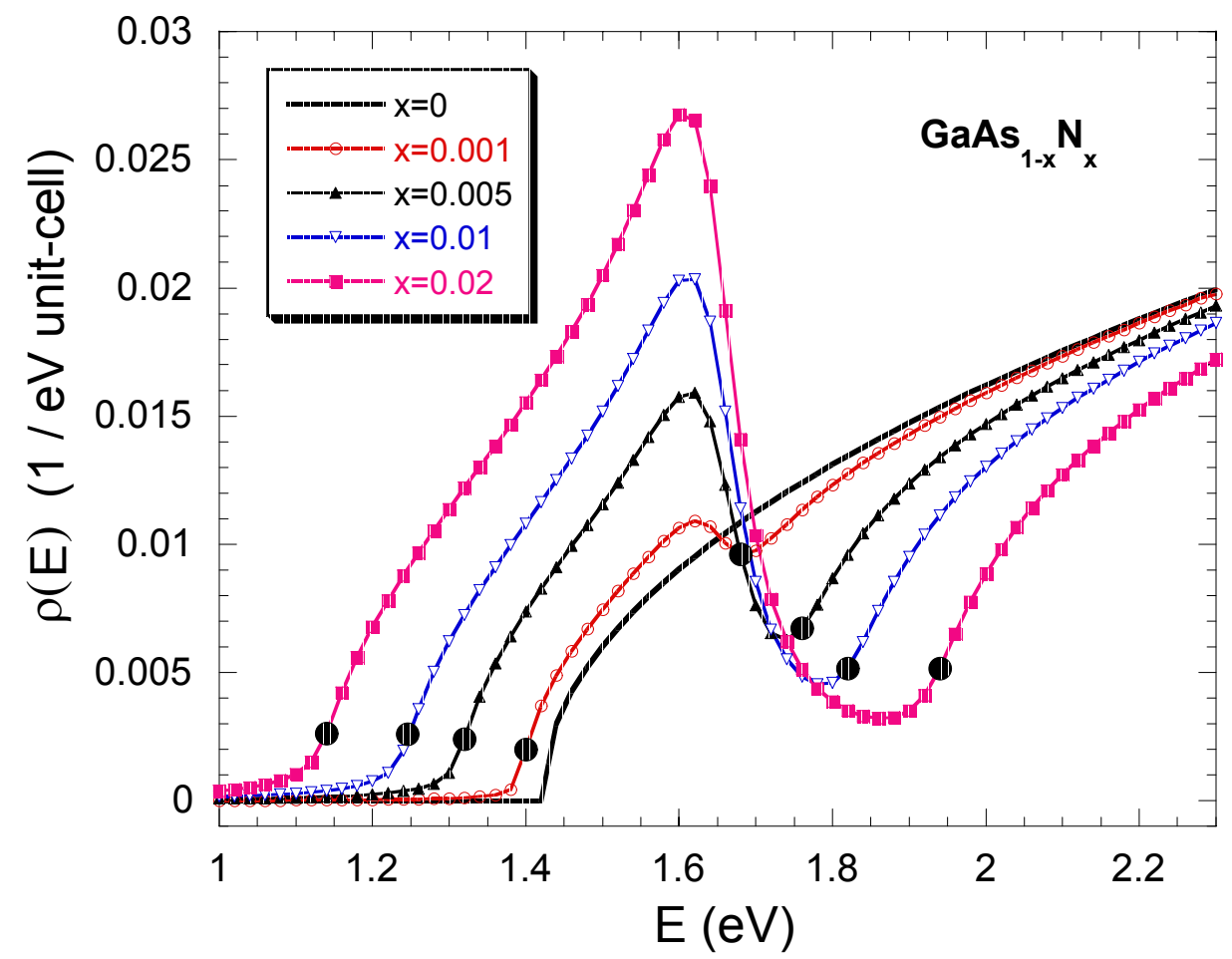

Fig. 2 of 3

J. Wu et. al. 


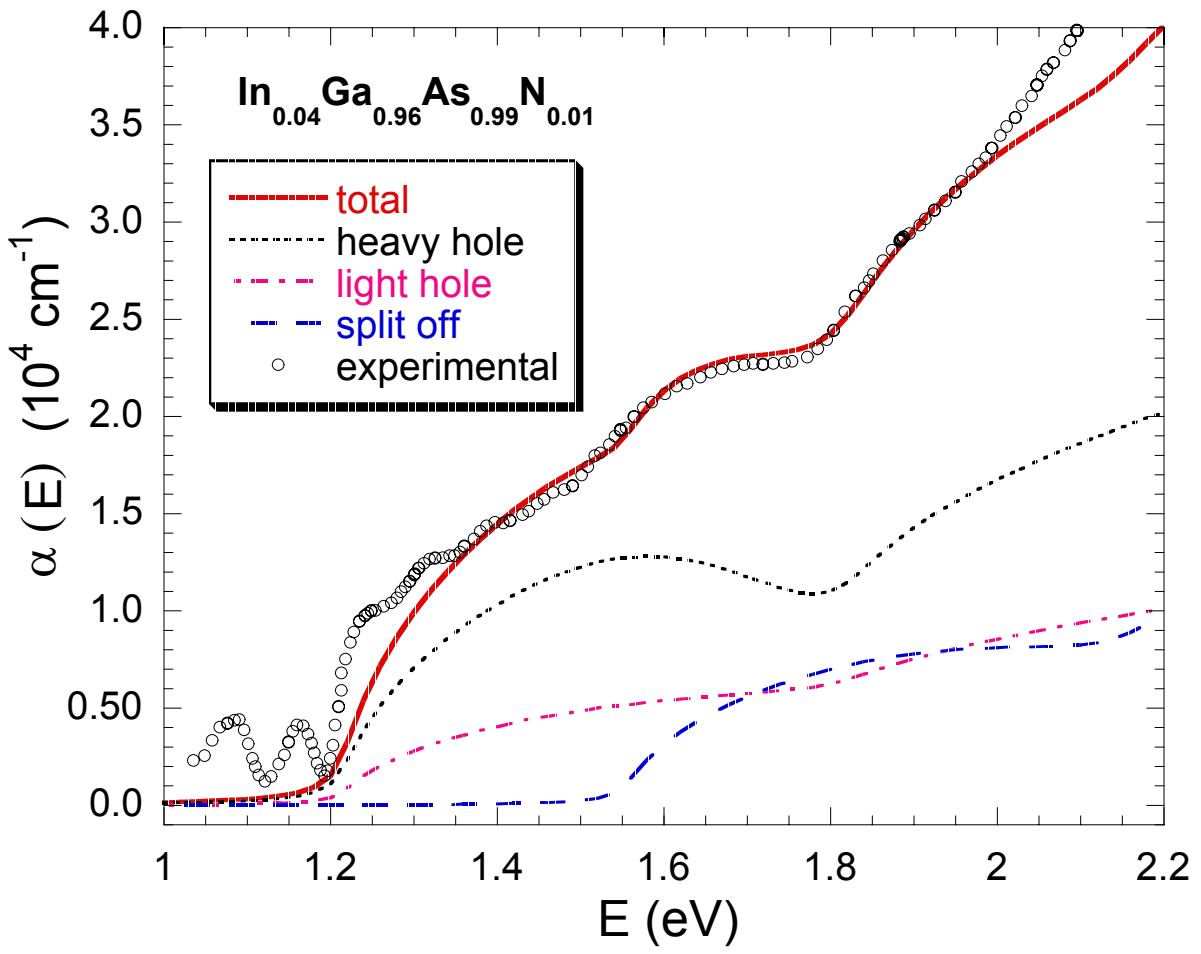

Fig. 3 of 3

J. Wu et. al. 